\title{
Omental Transposition to the Chronically Injured Human Spinal Cord ${ }^{\star}$
}

\author{
Harry S. Goldsmith, M.D., Glenn Neil-Dwyer, M.S., F.R.C.S. and Lorice \\ Barsoum F.F.S., R.C.S. \\ From the Departments of Surgery and Neurosurgery Boston University School of \\ Medicine, Boston, USA and The Brook General Hospital, London.
}

The omentum has been used over the years for a variety of clinical problems. Recently it has been shown that placing the omentum on the brain and spinal cord can lead to an extensive development of vascular connections at the omental/CNS interface. It has been further shown that an enormous amount of blood can be delivered through these connections between the omentum and the brain in an amount sufficient to prevent cerebral infarction in spite of experimental occlusion of the middle cerebral artery. These findings have led to recent reports throughout the world (United States, Europe, Asia) showing benefits from omental placement to the human brain in patients with chronic brain damage resulting from cerebral infarction.

Success with omental transposition to the human brain has led to increasing interest in placing the omentum on to the human spinal cord. Recent publications from our laboratory have shown that placement of the omentum on to the injured spinal cord of cats allowed for subsequent improvement in neuroelectrical activity, as manifested by reproducible somatosensory evoked potentials, and, more importantly, in motor function.

Four tetraplegic patients were chosen to enter into a feasibility study to see if omental transposition to their spinal cord might result in clinical benefit. All the patients had well defined upper cervical cord lesions. The length of time from their injury was $21,30,72$ and 180 months respectively. All patients had little, if any, motor and sensory function below shoulder level.

The operation required surgical lengthening of the pedicled omentum followed by its placement into a subcutaneous tunnel created along the anterior chest wall up to and over the shoulder. An extensive cervical laminectomy was then performed followed by a wide opening of the dura. In all instances the cord showed changes consistent with previous trauma. The omentum was laid directly on to the underlying spinal cord.

Three of the four tetraplegic patients showed motor and sensory changes within

* Summary of paper read at the Scientific Meeting of the International Medical Society of Paraplegia in Edinburgh, Scotland, September 1985. 
the first few post-operative days; an observation that was considered of great interest especially in one patient who had been injured fifteen years previously. These changes have slowly progressed over the following months; some being striking.

It would appear that omental transposition to the chronic spinal cord injured patient is worthy of expanded clinical trials. 